\title{
Eosinophilia in a patient with ankylosing spondylitis treated with prolonged adalimumab only
}

Junhong $\mathrm{He}^{1}$, Jing Luo², Qiong Yang ${ }^{1}$, Fangfang Yuan²

${ }^{1}$ Department of Pharmacy, Ningbo No. 6 Hospital, Ningbo, China

2Department of Rheumatism and Immunology, Ningbo No. 6 Hospital, Ningbo, China

Submitted: 11 December 2021; Accepted: 18 February 2022

Online publication: 23 February 2022

Arch Med Sci 2022; 18 (2): 564-567

DOI: https://doi.org/10.5114/aoms/146793

Copyright (c) 2022 Termedia \& Banach

In the past decade, tumor necrosis factor- $\alpha$ (TNF- $\alpha$ ) antagonists have been used as the first-line treatment for autoimmune diseases, including rheumatoid arthritis, ankylosing spondylitis (AS), Crohn's disease, uveitis, inflammatory bowel diseases, and psoriasis [1, 2]. In the 1970s, TNF- $\alpha$ was used as an anti-tumor drug by Lloyd Old et al. [3]. TNF- $\alpha$ was subsequently found to be a multifunctional cytokine with important roles in acute and chronic inflammation, antitumor responses, infection, and various disease processes, such as dyslipidemia, insulin resistance, and autoimmune diseases [4, 5]. Adalimumab has been approved by the United States Food and Drug Administration. The high affinity and specificity of adalimumab contribute to its safety and efficacy in clinical use [6]. Common adverse effects of adalimumab include infection, leukopenia, elevated creatinine phosphokinase and transaminases, headache, and skin rash [7]. Since adalimumab was approved for use in 2002, eosinophilia has been rarely reported following adalimumab treatment. The classification scheme of eosinophilia is as follows: mild, $0.5 \times 10^{9}-1.5 \times$ $10^{9} /$; moderate, $1.5 \times 10^{9}-5.0 \times 10^{9} /$; ; and severe, > $5.0 \times 10^{9} / \mathrm{l}$ [8]. Eosinophils directly cause cell damage by releasing specific granule contents, thereby damaging tissue integrity [9]. Thus, excess eosinophils exacerbate severe cell damage.

We describe a case of eosinophilia in a patient with prolonged administration of adalimumab to highlight the importance of assessing the blood count, particularly eosinophils, during administration of this biological agent. Further, we explore the use of disease-modifying antirheumatic drugs (DMARDs) for treating eosinophilia resulting from treatment with adalimumab.

A 17-year-old male who presented with a 1-year history of swelling and pain in both heels, accompanied by a 9-month history of pain in the back and right hip, was admitted to our hospital on July 17, 2014. On admission, physical examination revealed that his spine had an abnormal physiological curvature. Bipedal heel swelling and tenderness were noted. In specific tests, the bilateral straight leg elevation test was negative, Patrick's test on the right was positive, pillow-wall distance was $5 \mathrm{~cm}$, Schober's test was $5 \mathrm{~cm}$, and finger-to-ground distance was $30 \mathrm{~cm}$. The muscle tone was normal, and bilateral tendon reflexes were elicited symmetrically without pathological reflexes.

Laboratory test results were as follows: human leukocyte antigen B27, positive; C-reactive protein, $15.6 \mathrm{mg} / \mathrm{l}$; and erythrocyte sedimentation rate, $64 \mathrm{~mm} / \mathrm{h}$. Based on the comprehensive clinical assessment, labo-

\author{
Corresponding author: \\ Fangfang Yuan \\ Department of \\ Rheumatism and \\ Immunology \\ Ningbo No. 6 Hospital \\ Ningbo, China \\ E-mail: 2063940547@qq.com
}


ratory tests, and imaging, the diagnosis of AS was established.

During hospitalization, the patient was administered adalimumab (40 mg) once every 2 weeks by subcutaneous injection after excluding relevant contraindications. Sulfasalazine tablets $(1.0 \mathrm{~g}$, twice per day) were administered orally along with doses of adalimumab. After 4 months of combination therapy with adalimumab and sulfasalazine, the patient's symptoms were significantly relieved. The patient decided to discontinue treatment with adalimumab and continued with sulfasalazine tablets (1.0 g, twice per day) orally to control his condition.

On January 30, 2015, the patient experienced swelling and pain in both heels. Considering disease recurrence, adalimumab (40 mg) was administered once every 2 weeks. Sulfasalazine tablets (1.0 g, twice per day) were administered orally along with adalimumab. After 3 months, the patient's condition improved. On January 23, 2017, sulfasalazine tablets and adalimumab were discontinued because of the patient's low globulin level of $13.9 \mathrm{~g} / \mathrm{l}$. After 6 months, the patient's globulin level returned to normal, and methotrexate tablets (10 mg, once per week) were administered orally. However, laboratory tests revealed an alanine aminotransferase level of $172 \mathrm{U} / \mathrm{l}$ and aspartate aminotransferase level of $68 \mathrm{U} / \mathrm{l}$ after administration of methotrexate tablets for 2 weeks. Thus, methotrexate tablets were immediately discontinued.

Subsequently, the patient was administered adalimumab (40 mg) only. After 1 week of treatment, routine blood tests showed an absolute eosinophil count of $0.66 \times 10^{9} /$, leading to discontinuation of adalimumab. At this time, the patient had fatigue and discomfort. Eosinophilia was diagnosed through bone marrow tests at the Department of Hematology, Ningbo First Hospital. Bone marrow gene detection showed no myeloid-related pathogenic mutations. In addition, fluorescence in situ hybridization and qualitative detection of the ETV6-PDGFR and FIP1L1/PDGFR $\alpha$ fusion gene were all negative. Given that the eosinophilia was unexplained, the clinicians did not treat the conditions discussed. The eosinophilia persisted until May 11, 2019 (Table I).

Eosinophils, a type of human leukocyte, are extremely important in immune and allergic reactions. The physiological activity of eosinophils has been observed in multiple organs and tissues [10]. Eosinophils present antigens for killing bacteria and parasites and play key roles in inducing and maintaining chronic inflammation and tissue fibrosis [11]. Thus, the causes of eosinophilia are diverse. Hypereosinophilic syndrome is associated with multiple molecular defects in PCM1-JAK2, FGFR 1, PDGFR $\alpha$, and PDGFR 3 . Tyrosine kinase activity is abnormally increased in these genetic mutations, leading to eosinophil overproduction. Differential diagnoses for eosinophilia include the primary considerations of hematologic malignancies, such as acute and chronic myeloid leukemia, and systemic mastocytosis. Eosinophilia can occur in inflammatory and immune diseases [12]. In allergies and infections, clonal or phenotypically abnormal T cells secrete the cytokine, IL-5, which can cause eosinophilia if present in excess amounts [13]. Pulmonary insufficiency and disseminated cryptococcosis are other factors associated with an increased eosinophil count [14].

Drug-induced eosinophilia is typically transient, mild, or even asymptomatic. In most cases, eosinophils return to normal levels in patients after drug withdrawal and those with severe symptoms

Table I. Relationship between duration of the drug therapy and eosinophilia

\begin{tabular}{|lcccc|}
\hline Time & Adalimumab & Sulfasalazine & Methotrexate & Eosinophils \\
\hline $2014.7 .14-2014.11 .14$ & + & + & - & Normal \\
\hline $2014.11 .15-2015.1 .29$ & - & + & - & Normal \\
\hline $2015.1 .30-2017.1 .22$ & + & + & - & Normal \\
\hline $2017.1 .23-2017.7 .22$ & - & - & - & Normal \\
\hline $2017.7 .23-2017.8 .6$ & + & - & + & Normal \\
\hline $2017.8 .7-2018.12 .1$ & + & - & - & Normal \\
\hline 2018.12 .8 & + & - & - & $0.66 \times 10^{9} / /$ \\
\hline 2018.12 .15 & - & - & - & $1.33 \times 10^{9} / /$ \\
\hline 2018.12 .22 & - & - & - & $1.78 \times 10^{9} / /$ \\
\hline 2019.1 .29 & - & - & - & $2.08 \times 10^{9} / /$ \\
\hline 2019.3 .2 & - & - & - & $1.05 \times 10^{9} / /$ \\
\hline 2019.4 .17 & - & - & - & $1.05 \times 10^{9} / /$ \\
\hline 2019.5 .11 & - & - indicates that the drug was being used by the patient; “" indicates that the drug was discontinued. & Normal \\
\hline
\end{tabular}


recover quickly after symptomatic treatment. Few patients require corticosteroid treatment [15].

In this case, the patient was diagnosed with AS without a past history of asthma. The patient's living environment was stable. The cotton bedding sheets at home and school were regularly cleaned. There were no reported environmental allergens, such as flowers or pollen, and food allergen test results were negative. Fatigue and discomfort occurred after eosinophilia. Environmental and dietary factors were screened, and the possibility of hematological diseases and malignant tumors were excluded (Table II A). Thus, drug-induced eosinophilia was highly suspected. The relevant drugs were not administered within half a year before the occurrence of eosinophilia; after discontinuing the drug, the eosinophil level gradually decreased and returned to normal levels. This effect was suspected to be related to adalimumab treatment. The causality term was 'probable/likely' using the World Health Organization-Uppsala Monitoring Centre (WHO-UMC) scale (Table II B). The Naranjo Algorithm resulted in 'probable'. In addition, we observed that the patient's eosino- phils continued to rise to $2.08 \times 10^{9} / \mathrm{l}$ a month after discontinuation of adalimumab. We speculate that this phenomenon was due to the fact that it took the patient a month to clear the remaining adalimumab from the body, given that the half-life of a single intravenous dose of adalimumab ranges from 15 to 19 days [16].

Although cases are rare, treatment with TNF- $\alpha$ antagonists has been reported to be associated with eosinophilia in clinical scenarios [17]. These cases suggest the involvement of unknown mechanisms that must be explored. Compared with previous cases, our case was unique because the increase in eosinophils did not occur immediately after adalimumab administration, but rather only after the patient discontinued methotrexate or sulfasalazine because of his medical condition. When methotrexate or sulfasalazine was combined with adalimumab, the patient did not develop eosinophilia. The reasons for using combination drug therapy were as follows: first, considering the poor prognosis of the patient, DMARDs were added to the adalimumab treatment; and second, considering the cost of the

Table II. A - Screening for causes of eosinophilia. B - WHO-UMC causality categories of the case

\begin{tabular}{|c|c|c|}
\hline A. Item & & Eosinophils \\
\hline \multirow[t]{4}{*}{ Environment } & Pollen allergy & Negative \\
\hline & Food allergy & Negative \\
\hline & Parasite exposure history & Negative \\
\hline & Ultraviolet allergy & Negative \\
\hline \multirow[t]{6}{*}{ Disease } & History of the tumor & Negative \\
\hline & History of blood disorders & Negative \\
\hline & History of bone marrow-related diseases & Negative \\
\hline & Virus/fungus/bacterial infection & Negative \\
\hline & History of skin diseases & Negative \\
\hline & History of asthma & Negative \\
\hline \multirow[t]{10}{*}{ Laboratory test } & ESR & Normal \\
\hline & CRP & Normal \\
\hline & FIP1L1/PDGFR $\alpha$ & Negative \\
\hline & ETV6-PDGFR & Negative \\
\hline & Hepatitis series & Normal \\
\hline & Anti-nuclear antibody & Negative \\
\hline & Rheumatoid factor & Negative \\
\hline & Anti-cyclic citrulline peptide antibody & Normal \\
\hline & Thyroid function & Normal \\
\hline & T-SPOT tuberculosis tests & Normal \\
\hline \multicolumn{3}{|l|}{ B } \\
\hline \multirow[t]{4}{*}{ Probable/Likely } & \multicolumn{2}{|c|}{ Event or laboratory test abnormality, with reasonable time relationship to drug intake } \\
\hline & \multicolumn{2}{|c|}{ Unlikely to be attributed to disease or other drugs } \\
\hline & \multicolumn{2}{|c|}{ Response to withdrawal clinically reasonable } \\
\hline & \multicolumn{2}{|c|}{ Rechallenge not required } \\
\hline
\end{tabular}

$C R P-C$-reactive protein, $E S R$ - erythrocyte sedimentation rate. 
biological agent, we planned to reduce the use of adalimumab in later stages. Methotrexate is a folic acid antagonist that can reduce the activity of adenosine synthetase, inhibit the synthesis of DNA, and plays an anti-inflammatory and immunosuppressive role. Currently, methotrexate is commonly used to treat rheumatism. Sulfasalazine is a sulfanilamide drug with antibacterial, anti-inflammatory, and anti-immune properties, and is recognized as a DMARD for treating rheumatoid arthritis. Methotrexate has been reported to cause pancytopenia and inhibit the synthesis of new purines in $\mathrm{T}$ lymphocytes, thus, affecting IL-5 levels [18]. Sulfasalazine reduces eosinophil activation. Both drugs may reduce the eosinophil count. Based on the medical history, the eosinophil-lowering effects of DMARDs may have been eventually offset by the adverse effects of adalimumab. Therefore, eosinophilia only appeared after discontinuation of combination therapy. However, no relevant reports exist to support this hypothesis, which should be further evaluated in basic research.

AS is a chronic and complex autoimmune disease affecting the spine and sacroiliac joints and has a prevalence of approximately $0.24 \%$ in the Chinese population. The high disability rate greatly affects the physical and mental health of patients. Presently, there is no cure for AS, and patients often require lifelong medication. DMARDs are used regularly in AS to treat several inflammatory conditions; however, they appear to have little or no effect on patients with axial AS. Thus, the emergence of anti-TNF drugs gives patients hope. Evidence suggests that anti-TNFs are the most effective drugs for treating AS. However, because of its high cost and adverse reactions, some patients must discontinue adalimumab treatment, despite its good therapeutic effects.

In conclusion, this case provides insight into the combined use of DMARDs and adalimumab, which can lower costs and leads to fewer adverse reactions. Moreover, we reported this case to improve our understanding of adverse reactions related to adalimumab. This rare case provides a foundation for further treatment options for AS.

\section{Acknowledgments}

We thank the patient and his parents for participating in this study. We thank all of our colleagues in the hospital who have been involved in the patient's care and research. We would like to thank Editage (www.editage.cn) for English language editing.

\section{Conflict of interest}

The authors declare no conflict of interest.

\section{References}

1. Fleischmann RM, Genovese MC, Enejosa JV, et al. Safety and effectiveness of upadacitinib or adalimumab plus methotrexate in patients with rheumatoid arthritis over 48 weeks with switch to alternate therapy in patients with insufficient response. Ann Rheum Dis 2019; 78: 1454-62.

2. LaMattina KC, Goldstein DA. Adalimumab for the treatment of uveitis. Expert Rev Clin Immunol 2017; 13: 181-8.

3. Carswell EA, Old LJ, Kassel RL, et al. An endotoxin-induced serum factor that causes necrosis of tumors. Proc Natl Acad Sci USA 1975; 72: 3666-70.

4. Cawthorn WP, Sethi JK. TNF-alpha and adipocyte biology. FEBS Lett 2008; 582: 117-31.

5. Palladino MA, Bahjat FR, Theodorakis EA, et al. AntiTNF-alpha therapies: the next generation. Nat Rev Drug Discov 2003; 2: 736-46.

6. Poddubnyy D, Rudwaleit M. Efficacy and safety of adalimumab treatment in patients with rheumatoid arthritis, ankylosing spondylitis and psoriatic arthritis. Exp Opin Drug Safety 2011; 10: 655-73.

7. Ellis CR, Azmat CE. Adalimumab. StatPearls, StatPearls Publishing. Copyright (c) 2021, StatPearls Publishing LLC., Treasure Island (FL), 2021.

8. Price DB, Rigazio A, Campbell JD, et al. Blood eosinophil count and prospective annual asthma disease burden: a UK cohort study. Lancet Respir Med 2015; 3: 849-58.

9. Persson T, Calafat J, Janssen H, et al. Specific granules of human eosinophils have lysosomal characteristics: presence of lysosome-associated membrane proteins and acidification upon cellular activation. Biochem Biophys Res Commun 2002; 291: 844-54.

10. Berek C. Eosinophils: important players in humoral immunity. Clin Exp Immunol 2016; 183: 57-64.

11. Ramirez GA, Yacoub MR, Ripa M, et al. Eosinophils from physiology to disease: a comprehensive review. BioMed Res Int 2018; 2018: 9095275.

12. Cafone J, Ruffner MA, Spergel JM. The role of eosinophils in immunotherapy. Curr Allergy Asthma Rep 2020; 20: 1.

13. Klion AD, Ackerman SJ, Bochner BS. Contributions of eosinophils to human health and disease. Ann Rev Pathol 2020; 15: 179-209.

14. Woodruff PG, Khashayar R, Lazarus SC, et al. Relationship between airway inflammation, hyperresponsiveness, and obstruction in asthma. J Allergy Clin Immunol 2001; 108: 753-8.

15. Shiohara T, Kano Y. Drug reaction with eosinophilia and systemic symptoms (DRESS): incidence, pathogenesis and management. Exp Opin Drug Safety 2017; 16: 139-47.

16. Weisman MH, Moreland LW, Furst DE, et al. Efficacy, pharmacokinetic, and safety assessment of adalimumab, a fully human anti-tumor necrosis factor-alpha monoclonal antibody, in adults with rheumatoid arthritis receiving concomitant methotrexate: a pilot study. Clin Ther 2003; 25: 1700-21.

17. Morales-Camacho RM, Prats-Martín C. Eosinophils with ring-shaped nuclei in a patient treated with adalimumab. Blood 2019; 133: 101.

18. Ajmani S, Preet Singh Y, Prasad S, et al. Methotrexate-induced pancytopenia: a case series of 46 patients. Int J Rheum Dis 2017; 20: 846-51. 